\title{
Breast cancer during pregnancy
}

Sandhya C. Acharya, MD; Aarati Shah, MD

Department of Clinical Oncology, National Academy of Medical Sciences (NAMS), Bir Hospital, Kathmandu, Nepal

\section{Corresponding Author}

Sandhya C Acharya, MD

Email: sandhyachap@gmail.com

Received 22 Nov 2018

Accepted 3 Dec 2018

\section{ABSTRACT}

Breast cancer during pregnancy is rare accounting for 1 in around 3000 pregnant women as per the American cancer society. According to hospital-based cancer registry of Nepal, breast cancer accounts for $9.2 \%$ of total cancer cases and pregnancy associated breast cancer accounts for $0.15 \%$ of the total breast cancer. Though rare, breast cancer is the most common type of cancer found during pregnancy and that too in advanced stage because of the hormonal interplay during pregnancy. Here we present a case of breast cancer during pregnancy which was treated successfully with chemotherapy, surgery, radiation therapy and is on hormonal treatment. Patient is on regular follow up for the last six years without evidence of recurrence of the disease, and the baby has achieved normal developmental milestones though exposed to chemotherapy in utero.

Key Words: Breast cancer, chemotherapy, cancer treatment, pregnancy

\section{Introduction}

Breast cancer during pregnancy is rare accounting for 1 in around 3000 pregnant women as per the American cancer society ${ }^{1}$. Breast cancer is one of the commonest cancers in Nepal accounting for $9.2 \%$ of total cancer cases with pregnancy associated breast cancer being $0.15 \%$ of the total breast cancer ${ }^{2}$. Though breast cancer during pregnancy is a very rare form, one should be cautious because it diagnosed in later stage, and usually with lymph node spread as compared to non-pregnant cases. There will be hormonal changes during pregnancy which increases the firmness and density of the breasts masking early detection of the lump and it can be easily attributed to the physiological breast changes due to the hormones in pregnancy ${ }^{3}$. Lumps may be readily missed when screenings are postponed due to pregnancy.

\section{Case Report}

A forty-year old lady presented with history of lump in her left breast for about a month which was progressively increasing in size with ulceration of the skin for two weeks. She also gave history of amenorrhea for four months. On examination, the lump was fixed to the chest wall with skin ulceration that bled on touch. Clinically, it was T4cN2 disease. Ultrasonography (USG) of left breast demonstrated $5.5 \times 6.0 \times 4.2 \mathrm{~cm}$ hypoechoic irregular mass consistent with BIRADS 4 classification with axillary nodes. A needle biopsy from the lump revealed invasive ductal carcinoma that was positive for estrogen and progesterone receptors and negative for HER2. At the same instance USG of abdomen and pelvis revealed intrauterine single live fetus of fourteen weeks gestational age. Since she had three live issues before, option for medical termination of the pregnancy (MTP) was given but the couple strongly denied MTP and agreed on cancer treatment. She was in the second trimester of pregnancy and was taken for neoadjuvant chemotherapy as it was T4c disease.

Upon detailed discussion, informed consent was obtained and treatment started following baseline investigations. She received six cycles of chemotherapy with FAC regimen (5-flurouracil, Doxorubicin, Cyclophosphamide). She was monitored intensively and before each cycle of 
chemotherapy USG was done to ensure fetal wellbeing. She could complete all cycles of chemotherapy without any complications. She was under regular consultation with an Obstetrician as she could go into pre-term labor any time. She was taken up for elective LSCS three weeks after the completion of chemotherapy which coincided with 37 weeks of pregnancy. Full term, healthy, live single fetus of $2.48 \mathrm{~kg}$ with normal APGAR score was delivered. One month after the delivery of the baby she underwent modified radical mastectomy (MRM) of her left breast. Her postoperative periods were uneventful. Three weeks after the MRM she received adjuvant radiation therapy. She was then started on hormonal therapy as she had positive hormonal receptors. At six years of regular follow up there is no evidence of recurrence of the disease. The baby is achieving normal growth curve for his age.

\section{Discussion}

Pregnancy-associated breast cancer (PABC) is defined as breast cancer diagnosed during pregnancy or in the first postpartum year. The situation is complex dealing with two lives and maternal treatment is essential, though possibly harmful to the fetus. Because of limited data on this, physicians may be reluctant to take the challenge and that may pose risks to both the lives ${ }^{4}$. The patient's dilemma might be as difficult as that of the physician. Hence, a tailored approach to treatment is required with careful consideration given at all stages to the needs of the mother and risks to the fetus. Management is critically influenced by the stage of pregnancy maintaining medical and ethical consensus.

The incidence of breast cancer during pregnancy is expected to rise due to changing social structure of delayed marriage, and hence child birth. It is also projected to increase correlating with the increasing number of perimenopausal women diagnosed with breast cancer ${ }^{4-6}$.

Our patient at the diagnosis had T4cN2 disease as is common with the pregnant breast cancer patients. Pregnancy has complex interplay of hormones and due to the pregnancy related physiological changes, the diagnosis is usually delayed. Case series and case-controlled studies have demonstrated advanced stage at diagnosis of PABC and usually with lymph node metastasis compared to their non-pregnant counterpart ${ }^{4,7,8}$.
In the reported case, patient received standard anthracycline based neoadjuvant chemotherapy with FAC regimen as she was running second trimester of pregnancy. Literatures have shown that it is acceptable to administer anthracycline during second and third trimester of pregnancy with minimal fetal risk $^{7,9}$. Chemotherapy is contraindicated during first trimester of pregnancy and can be given relatively safely after the period of organogenesis. The most frequent undesired fetal outcome is premature delivery $4,6,7,9-12$.

Current guideline recommendations for breast cancer management during pregnancy is similar to that of non-pregnant patients ${ }^{13}$. Our patient was managed surgically with post-partum MRM of the left breast three weeks after completion of chemotherapy and therapeutically by hormonal, chemotherapy and radiation therapy. The patient is on sixth year of follow-up without evidence of disease recurrence. The baby has achieved normal developmental milestones.

Aviles et al. ${ }^{14}$ performed a cross-sectional study of pregnant women with hematologic malignancies and examined the outcomes of 84 children exposed to chemotherapy in utero. They found that the children's learning and educational performance were normal and no congenital, neurologic, psychologic, cardiac, or cytogenetic abnormalities or malignancies were noted. At the University of Texas MD Anderson Cancer Center, Hahn et al. ${ }^{9}$ evaluated pregnant breast cancer women who received neoadjuvant or adjuvant anthracycline based chemotherapy. In 2006 they reported initial 7 years follow-up of 52 children exposed to chemotherapy in utero and found no significant short-term complications for the majority of those children. Similarly, in 2014 Murthy et al. ${ }^{15}$ provided an eight-year update of prospective single institutional study comprising 82 children who were exposed to anthracycline in utero secondary to maternal breast cancer and found that the cohort of children exposed to chemotherapy in utero appeared to be doing well, with risk similar to that of the general population.

\section{Conclusion}

Pregnancy associated breast cancer is different because of its unique hormonal milieu. It should be treated aggressively according to the standards applicable in non-pregnant women. Fortunately, PABC is uncommon and should have 
multidisciplinary, patient tailored treatment with careful consideration given at all stages to the needs of the mother and risks to the mother/fetus. Management is critically influenced by the stage of pregnancy. All stages of management should be discussed in multidisciplinary team and clear treatment options should be sketched balancing the risks and benefit to both the mother and the fetus so that breast cancer during pregnancy is no longer "poena magna" as stated by Pavlidis ${ }^{16}$.

\section{References}

1. Byrd BF Jr, Bayer DS, Robertson JC, Stephenson SE Jr. Treatment of breast tumors associated with pregnancy and lactation. Ann Surg. 1962;155: 940-7.

2. National cancer registry programme, Hospital Based Cancer Registry - 10 yrs. consolidated report. B.P. Koirala Memorial Cancer Hospital: 2012. Available from: http://bpkmch.org.np/sites/default/files/ NCRP_10_Yrs_Consolidated_Report.pdf

3. C M Saunders, M Baum. Breast cancer and pregnancy: a review. J R Soc Med. 1993;86(3): 162-5.

4. Amant F, Deckers S, Van Calsteren K, Loibl S, Halaska $M$, Brepoels $L$, et al. Breast cancer in pregnancy: recommendations of an international consensus meeting. Eur J Cancer. 2010;46(18):3158-68.c

5. Ranstam J, Janzon L, Olsson H. Rising incidence of breast cancer among young women in Sweden. $\mathrm{Br}$ J Cancer. 1990;61(1):120-2.

6. Loibl S, von Minckwitz G, Gwyn K, Ellis P, Blohmer $J U$, Schlegelberger B, et al. Breast carcinoma during pregnancy. International recommendations from an expert meeting. Cancer 2006;106(2):237-46.
7. Ring $A E$, Smith $I E$, Jones $A$, Shannon $C$, Galani $E$, Ellis PA. Chemotherapy for breast cancer during pregnancy: an 18-year experience from five London teaching hospitals. J Clin Oncol. 2005;23(18): 4192-7.

8. Shousha S. Breast carcinoma presenting during or shortly after pregnancy and lactation. Arch Pathol Lab Med. 2000;124(7):1053-60.

9. Hahn KM, Johnson $\mathrm{PH}$, Gordon $\mathrm{N}$, Kuerer $\mathrm{H}$, Middleton $L$, Ramirez $M$, et al. Treatment of pregnant breast cancer patients and outcomes of children exposed to chemotherapy in utero. Cancer. 2006;107(6):1219-26.

10. Germann N, Goffinet F, Goldwasser F. Anthracycline during pregnancy: embryo fetal outcome in 160 patients. Ann Oncol. 2004;15(1):146-50.

11. Ring $A E$, Smith $I E$, Ellis PA. Breast cancer and pregnancy. Ann Oncol 2005;16 (12):1855-60.

12. Dziadek O, Singh P. Breast Cancer in Pregnancy. Case Rep Womens Health. 2014;3(4):7-9.

13. Navrozoglou I, Vrekoussis T, Kontostolis E, Dousias V, Zervoudis S, Stathopoulos EN, et al. Breast cancer during pregnancy: A mini-review. Eur J Surg Oncol. 2008;34(8):837-43.

14. Avilés $A$, Neri $N$. Hematological malignancies and pregnancy: a final report of 84 children who received chemotherapy in utero. Clin Lymphoma. 2001;2(3):173-7.

15. Murthy RK, Theriault RL, Barnett CM, Hodge S, Ramirez MM, Milbourne A, et al. Outcomes of children exposed in utero to chemotherapy for breast cancer. Breast Cancer Res. 2014;16(6): 500.

16. Cancer and pregnancy: poena magna, not anymore. Eur J Cancer. 2006;42(2):126 -40. 